\title{
RESEARCH
}

\section{Interaction of BRAF-induced ETS factors with mutant TERT promoter in papillary thyroid cancer}

\author{
Young Shin Song1,2, Seong-Keun Yoo ${ }^{3}$, Hwan Hee Kim4, Gyeongseo Jung4, Ah-Reum Oh, Ji-Young Cha5, Su-jin Kim6, \\ Sun Wook Cho', Kyu Eun Lee ${ }^{3,6}$, Jeong-Sun Seo ${ }^{3}$ and Young Joo Park ${ }^{1,3,4}$ \\ 'Department of Internal Medicine, Seoul National University College of Medicine, Seoul, Korea \\ 2Department of Internal Medicine, CHA Bundang Medical Center, CHA University, Seongnam, Korea \\ ${ }^{3}$ Genomic Medicine Institute, Medical Research Center, Seoul National University, Seoul, Korea \\ ${ }^{4}$ Center for Medical Innovation, Seoul National University Hospital, Seoul, Korea \\ ${ }^{5}$ Department of Biochemistry, Lee Gil Ya Cancer and Diabetes Institute, GAIHST, Gachon University College of Medicine, Incheon, Korea \\ ${ }^{6}$ Department of Surgery, Seoul National University College of Medicine, Seoul, Korea
}

Correspondence should be addressed to Y J Park: yjparkmd@snu.ac.kr

\begin{abstract}
Synergistic effects of BRAFV600E and TERT promoter mutations on the poor clinical outcomes in papillary thyroid cancer (PTC) have been demonstrated. The potential mechanism of this phenomenon has been proposed: MAPK pathway activation by the $B R A F^{V 600 E}$ mutation may upregulate E-twenty six (ETS) transcription factors, increasing TERT expression by binding to the ETS-binding site generated by the TERT promoter mutation; however, it has not yet been fully proven. This article provides transcriptomic insights into the interaction between BRAFV600E and TERT promoter mutations mediated by ETS factors in PTC. RNA sequencing data on 266 PTCs from The Cancer Genome Atlas and 65 PTCs from our institute were analyzed for gene expression changes and related molecular pathways, and the results of transcriptomic analyses were validated by in vitro experiments. TERT mRNA expression was increased by the coexistence of BRAFV60E and TERT promoter mutations (fold change, 16.17 ; $q$-value $=7.35 \times 10^{-12}$ vs no mutation). In the ETS family of transcription factors, ETV1, ETV4 and ETV5 were upregulated by the $B R A F^{V 600 E} / M A P K$ pathway activation. These BRAFV60E-induced ETS factors selectively bound to the mutant TERT promoter. The molecular pathways activated by BRAFV600E were further augmented by adding the TERT promoter mutation, and the pathways related to immune responses or adhesion molecules were upregulated by TERT expression. The mechanism of the synergistic effect between BRAFV600E and TERT promoter mutations on cancer invasiveness and progression in PTC may be explained by increased TERT expression, which may result from the BRAF-induced upregulation of several ETS transcription factors.
\end{abstract}

Key Words
- $T E R T$ promoter mutation
- $B R A F^{V 600 E}$ mutation
- thyroid cancer
- prognosis
- mechanism

Endocrine-Related Cancer (2019) 26, 629-641

\section{Introduction}

Telomerase reverse transcriptase (TERT) has a canonical role maintaining telomere length and the nontelomeric function, which can regulate the expression of various genes involved in cell proliferation and cellular signaling, and both canonical and noncanonical roles may contribute to tumorigenesis and cancer progression c) 2019 Society for Endocrinology Published by Bioscientifica Ltd. Printed in Great Britain 
(Low \& Tergaonkar 2013, Li \& Tergaonkar 2014). The promoter region of the TERT gene has two hot spots susceptible to a point mutation: chr5, 1,295,228 C>T and 1,295,250 C>T, and both mutations create a binding motif for the ETS family of transcription factors (Horn et al. 2013, Huang et al. 2013).

TERT promoter mutation in thyroid cancer was first reported in 2013 (Liu et al. 2013) and has been shown to be associated with not only aggressive clinicopathological features but also poor prognosis (Liu et al. 2016). In particular, TERT promoter mutation is associated with the BRAFV600E mutation (Liu et al. 2013), which is the most common alteration and is also related to aggressiveness of papillary thyroid cancer (PTC) (Xing 2010, Song et al. 2015). When they coexist, this genetic duet exerts a synergistic negative effect on clinical outcomes (Xing et al. 2014, Song et al. 2016, Liu et al. 2017, Moon et al. 2017). The potential mechanism behind the synergism between the two mutations has been proposed: MAPK pathway activation by $B R A F^{V 600 E}$ may upregulate ETS transcription factors, which increase TERT expression by binding to the ETS-binding site generated by the TERT promoter mutation (Xing et al. 2014). Nonetheless, this mechanism of the synergistic duet in thyroid cancer has not been fully proven.

Recent advances in next-generation sequencing (NGS) started to provide important insights for our understanding of the molecular pathogenesis of thyroid cancers (Cancer Genome Atlas Research Network 2014, Landa et al. 2016, Yoo et al. 2016). Therefore, we investigated the molecular mechanism of the synergistic effects of $B R A F^{V 600 E}$ and TERT promoter mutations in PTC by analyzing the transcriptomic changes according to the mutational status using the NGS database of The Cancer Genome Atlas (TCGA) and validated the findings with our data (Yoo et al. 2016).

\section{Materials and methods}

\section{Data acquisition and selection of patients}

From the genomic data on anonymized patients with PTC that are available from TCGA data portal (https://portal. gdc.cancer.gov/), we downloaded the data on clinical information, somatic mutations, mRNA expression and DNA methylation in September 2016. Whole exome sequencing and mRNA sequencing were performed on the Illumina HiSeq 2000 platform. A total of 387 samples had the TERT promoter sequencing results from either Illumina MiSeq or whole genome sequencing. To remove possible influences from driver mutations or fusions other than BRAFV600E or TERT promoter mutation, 64 cases with other driver mutations and 57 cases with any driver fusions were then excluded (Supplementary Table 1, see section on supplementary data given at the end of this article). The driver genetic alterations were defined as the significantly mutated genes and driver fusions, according to definitions in a TCGA study (Cancer Genome Atlas Research Network 2014). Finally, 266 patients with PTC were included in the present analysis, and we classified them by mutational status: 41 patients negative for the driver genetic alterations including BRAFV600E and TERT promoter mutations (no mutation), 196 patients with the $B R A F^{V 600 E}$ mutation only (BRAF-only), 2 patients with a TERT promoter mutation only (TERT only) and 27 patients with coexistent BRAFV600E and TERT promoter mutations (BRAF+TERT) (Supplementary Fig. 1). For the comparison of differentially expressed genes (DEGs), the TERT-only group was excluded because of the small number of subjects in this group.

As a validation set of genomic analysis, among 125 PTC samples having RNA sequencing data from our previous research (hereafter, SNU database; Yoo et al. 2016), 24 patients with other driver mutations and 22 with other driver fusions were excluded. In addition to the previous study, we performed Sanger sequencing for the detection of TERT promoter mutation by a previously described method (Song et al. 2016). A total of 65 tumor DNA samples available in quantities enough to analyze TERT promoter mutations were included in the final analysis: 9 patients in the no-mutation group, 51 patients in the $B R A F$-only group, 1 patient in the TERT-only group and four patients in the BRAF+TERT group (Supplementary Fig. 1). This study's protocol was approved by the Institutional Review Board of Seoul National University Hospital, in accordance with the Declaration of Helsinki (approval ID: H-1108-041-372). Written informed consent was obtained from each subject.

\section{Gene expression profiling and DEG analysis}

Using counted numbers of reads aligned to each gene, we normalized them by the regularized log (rlog) transformation method of DESeq2 (Love et al. 2014). The DEGs were determined by DESeq 2 to have $q$-value $<0.05, \mid \log _{2}$ (fold change) $\mid \geq 1$, and baseMean $\geq 100$ and were illustrated using volcano plots which show the magnitude and the statistical significance of differential translation for each gene. The calculated $P$ values were adjusted to $q$-values for multiple testing using 
the Benjamini-Hochberg correction. To construct a heatmap, the centered rlog values were applied to the K-means clustering algorithm using cluster 3.0 (de Hoon et al. 2004). To identify molecular pathways that were significantly enriched in DEGs, we searched the Kyoto Encyclopedia of Genes and Genomes pathway database (Ogata et al. 1999). For comparison of mRNA expression among groups, normalized count data were subjected to the analyses.

\section{Cell culture}

Nthy-ori 3-1 is an immortalized thyroid follicular epithelial cell line derived from normal adult thyroid tissue, and Nthy-ori 3-1 cell clones expressing either wild-type $B R A F$ (Nthy/WT) or mutant $B R A F$ (Nthy/V600E) were established (Kim et al. 2017). BCPAP and MDA-T32, the human PTC cell lines harboring both BRAFV600E and TERT promoter mutations, were kindly provided by Dr Minho Shong (Chungnam National University, Daejon, Korea) and purchased from the American Type Culture Collection, respectively. KTC-2, FRO, SW1736 and $8505 \mathrm{C}$ are the human anaplastic thyroid cancer cell lines harboring BRAFV600E and TERT promoter mutations. The former two cell lines were kind gifts from Dr June-Key Chung (Seoul National University College of Medicine, Seoul, Korea), and SW1736 and 8505C cells were kindly provided by Dr Yoon Woo Koh (Yonsei University College of Medicine, Seoul, Korea) and Dr Seong Jin Lee (Hallym University College of Medicine, Chuncheon, Korea), respectively. KTC-2 cells were cultured in the RPMI 1640 medium supplemented with $5 \%$ of fetal bovine serum and all other cells were cultured in the RPMI 1640 medium supplemented with 10\% of fetal bovine serum, and grown at $37^{\circ} \mathrm{C}$ in a humidified atmosphere containing $5 \%$ of $\mathrm{CO}_{2}$.

\section{RT-PCR analysis}

The mRNA from the cultured Nthy/WT and Nthy/V600E cells was extracted by means of the TRIzol Reagent (Invitrogen). First-strand cDNA was synthesized from $1 \mu \mathrm{g}$ of total RNA with the Superscript II Reverse Transcriptase Kit (Invitrogen). Human TERT, EHF, ELF3, ETV1, ETV4 and ETV5 gene expression levels were quantified by PCR with the SYBR Green PCR master mix (Takara) on a StepOne Plus real-time PCR system (Applied Biosystems). The primers are listed in Supplementary Table 2. The reaction was conducted under the thermal cycling conditions as follows: $2 \mathrm{~min}$ at $95^{\circ} \mathrm{C}$, and then 40 cycles of $15 \mathrm{~s}$ at $95^{\circ} \mathrm{C}$ and 1 minute at $60^{\circ} \mathrm{C}$.

BCPAP cells were treated with PLX4720 (1 $1 \mu \mathrm{M}$, Selleckchem), a BRAF inhibitor, or PD98059 $(10 \mu \mathrm{M}$, Cell Signaling Technology), an ERK inhibitor. After $24 \mathrm{~h}$ of treatment, the cells were harvested, and mRNAs were extracted for the analysis of expression levels as described above.

\section{Western blot analysis}

BCPAP cells were treated with PLX4720 (0.1, 0.5, 1 and $10 \mu \mathrm{M})$, a $B R A F$ inhibitor, and the cell lysates were harvested at $24 \mathrm{~h}$. Proteins $(15 \mu \mathrm{g})$ were separated by $8 \%$ SDS-PAGE and transferred to nitrocellulose membranes. Membranes were incubated with primary antibodies overnight at $4^{\circ} \mathrm{C}$. The primary antibodies were pERK (1:1000, \#4370), ERK (1:2000, \#9102) from Cell Signaling Technology, and $\beta$-ACTIN (A5441) from Sigma-Aldrich. The secondary antibodies were anti-rabbit and anti-mouse. Bound primary antibodies were detected by incubation of the membranes with a secondary horseradish peroxidaseconjugated IgG antibody and visualized using Amersham Imager 600 (GE Healthcare).

\section{Chromatin immunoprecipitation assay}

The chromatin immunoprecipitation (ChIP) assay was performed as described previously (Jeong et al. 2011) using the EpiQuik Tissue Chromatin Immunoprecipitation kit (P-2002, EpiGentek, NY, USA). Briefly, Nthy-ori 3-1 $\left(3 \times 10^{6}\right.$ cells $)$, KTC-2 $\left(3 \times 10^{6}\right.$ cells $)$, BCPAP $\left(3 \times 10^{6}\right.$ cells $)$ and MDA-T32 $\left(3 \times 10^{6}\right.$ cells $)$ cells were cross-linked with $1 \%$ formaldehyde and blocked with $1.25 \mathrm{M}$ glycine solution. The cells were resuspended in CP3 lysis buffer containing a protease-inhibitor cocktail, and sonicated until the cross-linked chromatin was sheared to an average DNA fragment length of 200-300bp. Chromatin was immunoprecipitated with control IgG (\#2729; Cell Signaling technology), anti-ETV1 (sc-55581; Santa Cruz Biotechnology), anti-ETV4 (ARP32263_P050, Aviva Systems Biology, CA, USA) and anti-ETV5 (13011-1-AP, Proteintech, IL, USA), purified, and then analyzed by qPCR using a CFX384 Touch Real-Time PCR Detection System (Bio-Rad Laboratories, Inc.). KTC-2, BCPAP and MDA-T32 cells were treated with $1 \mu \mathrm{M}$ of PLX4720 for $24 \mathrm{~h}$. The primers were validated by analysis of template titration and dissociation curves. The primer sequences are listed in Supplementary Table 2. (c) 2019 Society for Endocrinology Published by Bioscientifica Ltd. Printed in Great Britain 


\section{Statistical analysis}

To compare the clinicopathological characteristics of subjects according to mutational status, Pearson's $\chi^{2}$ or Fisher's exact test (if the number was $<5$ ) was carried out for categorical variables and analysis of variance for continuous variables. The Mann-Whitney $U$ test was used to determine significance of difference between two groups in the assays of RT-PCR, western blotting and ChIP. The paired $t$ test was performed to compare differences in the expression levels of each common DEG between groups $B R A F$-only and $B R A F+T E R T$ or between groups BRAFVG00E mutation with TERT expression and without TERT expression. For multiple comparisons, a Bonferroni correction method was applied. Statistical significance was defined as two-sided $P$ values $<0.05$. All statistical analyses were performed in R v3.4.2 (www.r-project.org) or SPSS 23.0 (IBM Corp.).

\section{Results}

\section{Effects of the coexistence of BRAFV600E and TERT promoter mutations on poor clinicopathological outcomes in PTC}

The clinicopathological characteristics of subjects are presented in Table 1. Among the subjects from TCGA database, in comparison with the no-mutation group, the $B R A F$-only group was significantly associated with younger age at diagnosis and a higher proportion of conventional and tall-cell variant PTC, extrathyroidal extension and lymph node metastasis. Furthermore, the BRAF+TERT group was strongly associated with virtually all high-risk features such as older age at diagnosis, higher frequency of tall-cell variant PTC, larger tumor size, higher proportion of extrathyroidal extension, especially for moderate or advanced invasion, lymph node metastasis, mortality and higher stage or score of the prognosis prediction models, as compared to the no-mutation or BRAF-only group.

Among the subjects from the SNU database, as in TCGA database, the BRAF+TERT group showed high-risk clinicopathological characteristics, although there were some cases in which the small number of subjects in each group did not support statistical significance.

\section{Upregulated TERT mRNA expression in the coexistence of BRAFV600E and TERT promoter mutations}

To identify the mechanism behind the clinical aggressiveness of tumors harboring both BRAFV600E and TERT promoter mutations, we examined the mRNA expression level of TERT (Fig. 1). Compared with the no-mutation group, BRAF-only or TERT-only group did not show a significant difference in TERT expression (fold change, 2.30 and 1.54, respectively; $q$-value $=0.095$ and 0.886, respectively), whereas the BRAF+TERT group showed significantly higher TERT expression (fold change, 16.17; $q$-value $=7.35 \times 10^{-12}$; Fig. $\left.1 \mathrm{~A}\right)$. Nevertheless, it was unexpectedly found that the mRNA expression of TERT was detected in $25.5 \%$ of tumors in the BRAF-only group (50 of 196), and $14.6 \%$ of tumors in the no-mutation group (6 of 41; Fig. 1B). Similar to cases of the mutational coexistence, some clinicopathological characteristics, such as lymph node metastasis, recurrence and mortality, of the group 'coexistence of BRAFV600E mutation with TERT expression' showed greater aggressiveness as compared to the BRAFV600E mutation without TERT expression (Supplementary Table 3).

These results were similar for PTC tumors of the SNU dataset, although statistical significance was absent due to the limited number of subjects (Fig. 1C, D and Supplementary Table 4).

\section{Molecular mechanisms of upregulated TERT expression by the coexistence of BRAFV600E and TERT promoter mutations}

Transcription of TERT can be regulated various transcription factors recognizing their consensus sequence (Akincilar et al. 2016). To elucidate the possible mechanism of upregulated TERT expression for the combination of BRAFV600E and TERT promoter mutations, we studied the expression levels of representative transcriptional factors known to contain recognition sequences in the TERT promoter region (Akincilar et al. 2016, Ramlee et al. 2016): p53, p21, SP1, E2F, AP1, HIF1 and c-myc. There was no transcription factor gene whose expression was significantly changed (to satisfy the criteria of a DEG) by the presence of the BRAFV60oE mutation, except for AP1 (Supplementary Fig. 2 and Supplementary Table 5). AP1, which is a transcription factor composed of the c-Jun and c-Fos subunits, is known to regulate TERT expression, either activating or repressing TERT transcription (Ramlee et al. 2016), and the expression of $J U N$ and FOS was low in the BRAF+TERT group of both TCGA and SNU database; however, the BRAF-only group did not show significant changes.

Then, to evaluate the previously suggested mechanism, we determined whether the BRAFV00E mutation upregulates TERT by increasing ETS expression because a TERT promoter mutation creates a binding motif for ETS 


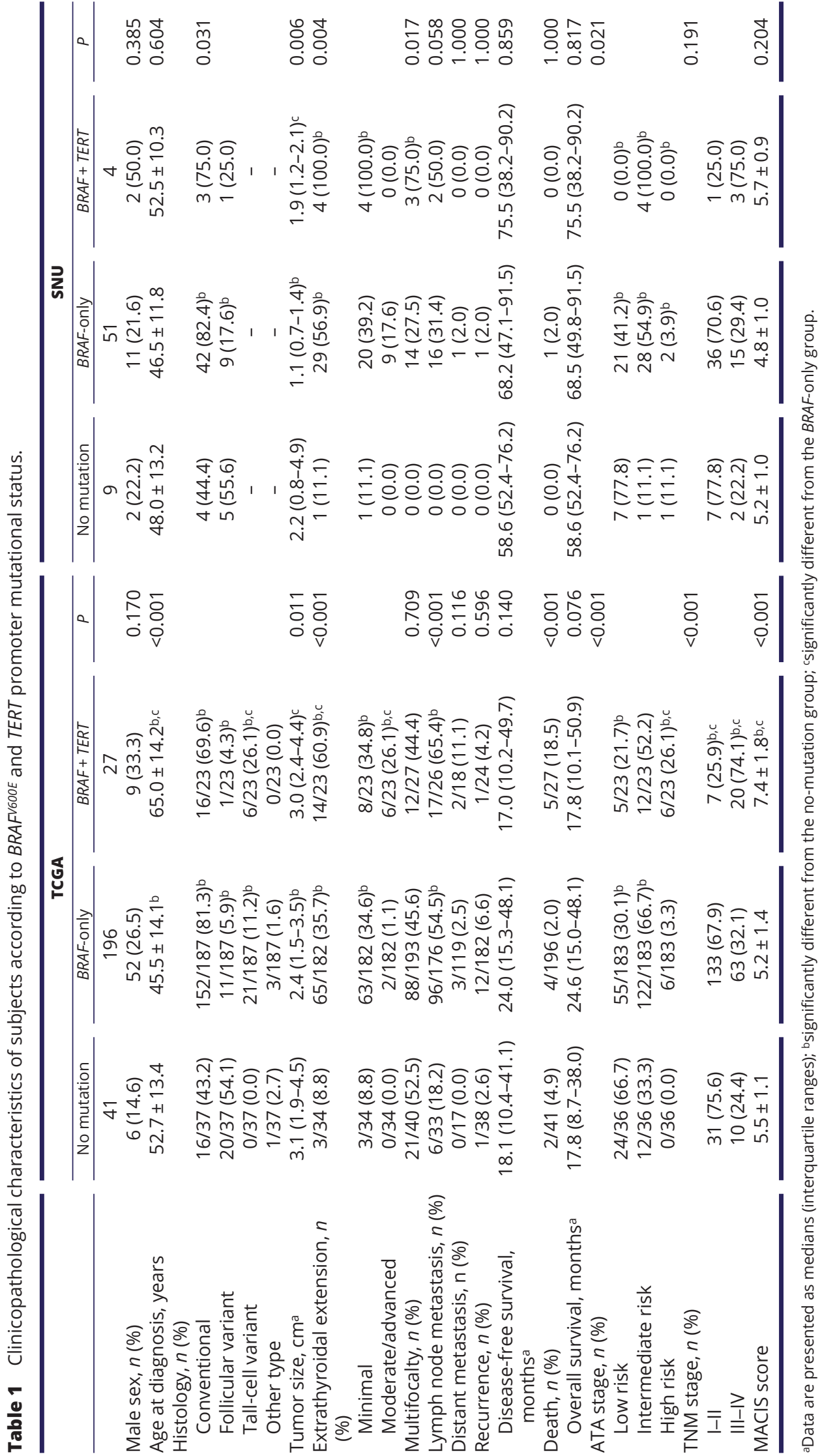


A

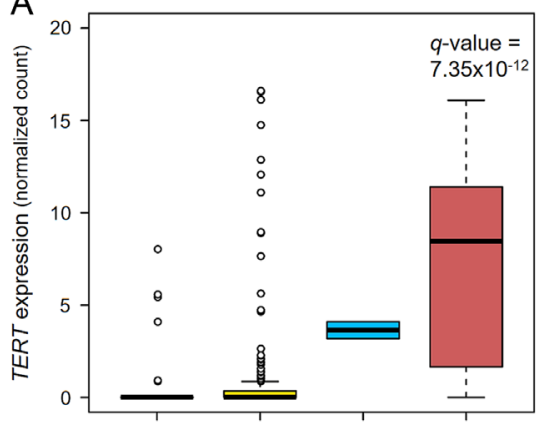

No mutation BRAF only TERT only BRAF+TERT

C

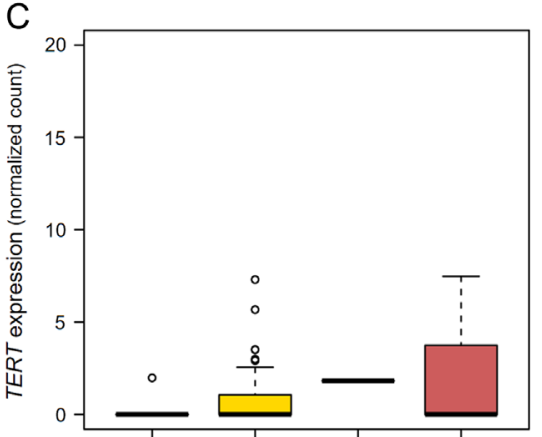

No mutation BRAF only TERT only BRAF+TERT
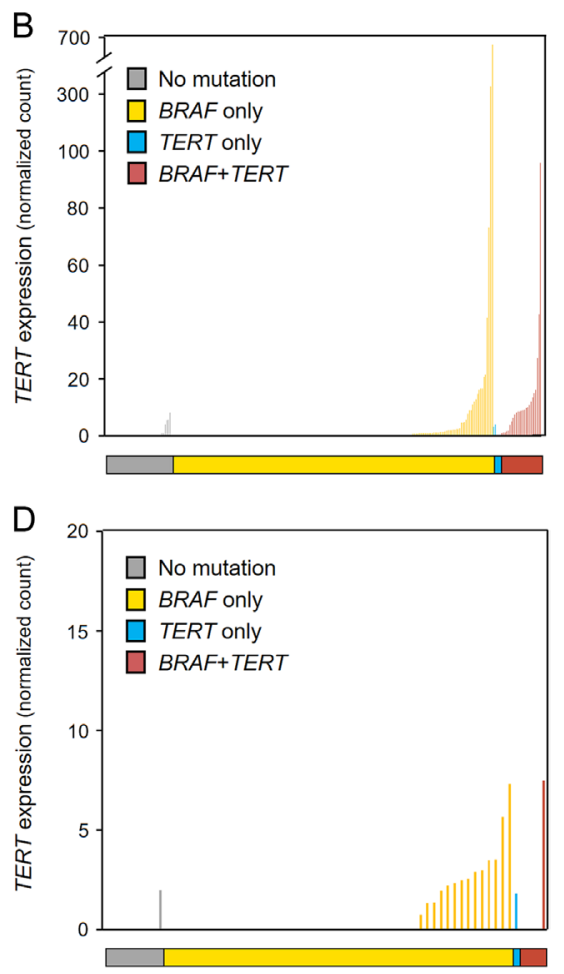

Figure 1

TERT mRNA expression according to mutational status. TERT mRNA expression levels from RNA sequencing data from TCGA ( $A$ and $B$ ) and SNU ( $C$ and $D$ ) database. ( $A$ and $C$ ) Median expression levels of TERT according to mutational status. ( $B$ and D) Each column represents an individual sample. transcription factors (Horn et al. 2013, Huang et al. 2013). In the whole ETS family, which consists of 28 genes (and 12 subfamilies), 2 genes (EHF and ELF3) of the ESE subfamily and three genes (ETV1, ETV4 and ETV5) of the PEA3 subfamily were significantly upregulated (in accordance with the criteria of upregulated DEGs) in the BRAF+TERT group compared to the no-mutation group. EHF, ELF3, ETV1 and ETV4 were similarly upregulated in the $B R A F$-only group compared with the no-mutation group (Fig. 2A, B, Supplementary Fig. 3A and Supplementary Table 6). When we analyzed PTC samples of the SNU dataset, similar results were obtained: EHF, ETV4 and ETV5 in the BRAF+TERT group and EHF, ETV7, ETV1, ETV4 and ETV5 in the BRAF-only group were found to be upregulated DEGs (Fig. 2C, Supplementary Fig. 3B and Supplementary Table 7).

\section{In vitro validation of changes in TERT and ETS expression by the BRAFV60E mutation and ETS binding to the mutant TERT promoter}

Among the possible mechanisms to regulate TERT expression, the ETS expression is consistently elevated in the presence of BRAFV600E mutation, which can be deduced a major mechanism of the effect of coexistent $B R A F^{V 600 E}$ and TERT promoter mutations.
To confirm the changes of TERT and ETS gene expression under the influence of the $B R A F^{V 600 E}$ mutation observed in the transcriptome data, we conducted in vitro experiments on overexpression or inhibition of BRAF. The expression of TERT in Nthy/V600E cells failed to show a statistically significant difference from Nthy/WT cells, although this expression was slightly higher in Nthy/V600E cells than in Nthy/WT cells (Fig. 3A and Supplementary Fig. 3C). Nonetheless, ETV1, ETV4 and ETV5 were significantly upregulated as expected, whereas ELF3 was downregulated in Nthy/V600E cells in contrast to the tissue expression pattern. The expressional changes of $E H F$ could not be evaluated because its expression was undetectable in both Nthy/WT and Nthy/V600E cells. We next treated the BRAF inhibitor (PLX4720) or ERK inhibitor (PD98059) to thyroid cancer cell lines harboring both BRAFV600E and TERT promoter mutations. The expression levels of genes TERT, ETV1, ETV4 and ETV5 were significantly lowered by BRAF and ERK inhibition, while ELF3 was upregulated (Fig. 3B and Supplementary Fig. 3D). In addition, the phosphorylation of ERK was suppressed by $B R A F$ inhibition in cells harboring both $B R A F^{V 600 E}$ and TERT promoter mutations (Fig. 3C).

Next, to demonstrate direct binding of ETV1, ETV4 and ETV5 to the TERT promoter in a mutation-dependent manner, ChIP assays were performed in the Nthy-ori 3-1
C 2019 Society for Endocrinology Published by Bioscientifica Ltd. Printed in Great Britain 
A $\square$ No mutation $\square B R A F$ only $\square B R A F+T E R T$

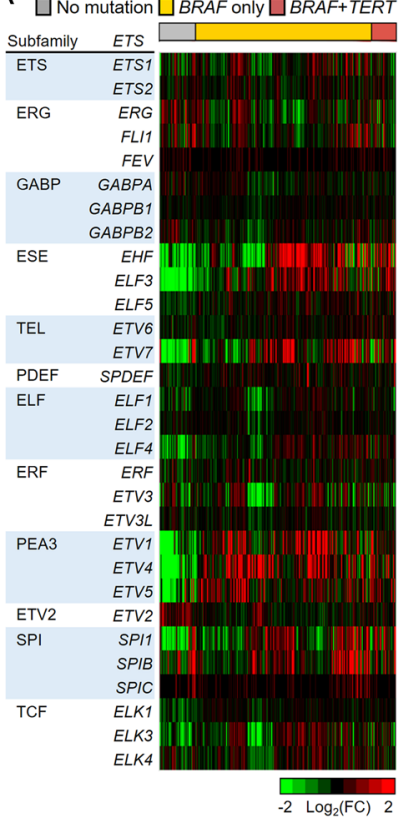

B

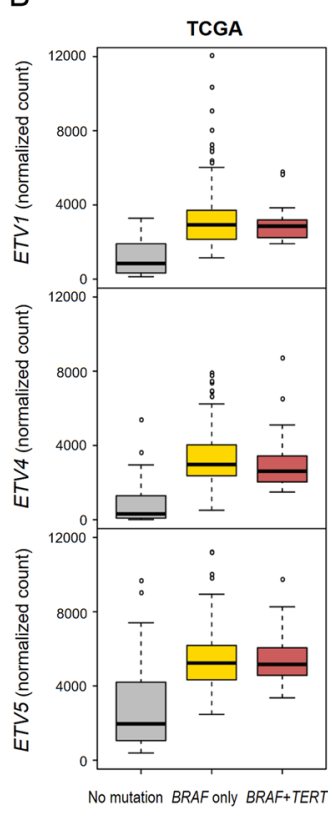

C

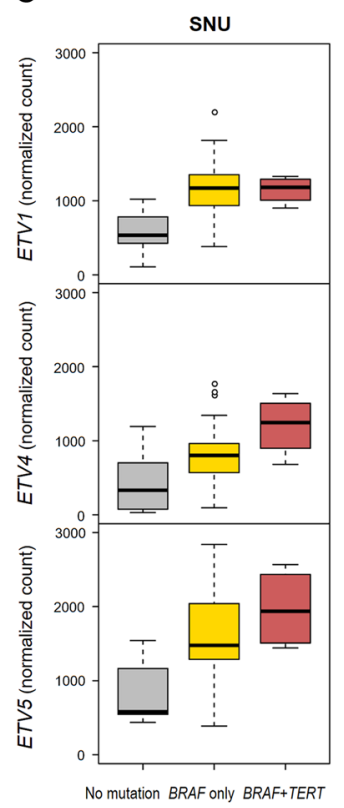

Figure 2

Upregulated expression of ETV1, ETV4, and ETV5 by the BRAFV600E mutation. (A) Heatmap of mRNA expression of $E T S$ according to mutational status in the samples from TCGA database. (B and C) Median expression levels of ETV1, ETV4 and ETV5 according to mutational status in TCGA (B) and SNU (C) database. cells (wild-type for BRAFV600E and TERT promoter) and KTC-2, BCPAP and MDA-T32 cells (harboring both $B R A F^{V 600 E}$ and TERT promoter mutations). We demonstrated a significant enrichment of ETV1, ETV4 and ETV5 binding at the TERT promoter region of KTC-2 cells, compared to Nthy-ori 3-1 cells (Fig. 3D). Moreover, we confirmed that the increased enrichment of these factors at the mutant $T E R T$ promoter was reduced by the $B R A F$ inhibitor, except for ETV1 in BCPAP and MDA-T32 cells (Fig. 3D).

\section{Changes in the intracellular signaling pathways by the coexistence of BRAFV600E and TERT promoter mutations}

To investigate whether the increased TERT expression by the coexistence of BRAFV600E and TERT promoter mutations causes some changes in the intracellular signaling pathways, which might reflect aggressive tumor behavior, we next compared the transcriptional profiles by mutational status. The results were illustrated in volcano plots, in which upregulated DEGs are denoted by red dots and downregulated DEGs by dark-green dots. Groups $B R A F$-only and $B R A F+T E R T$ showed a significant change in transcriptional profiles revealing a number of DEGs as compared to the no-mutation group (Fig. 4A and $B)$. On the other hand, there were few DEGs between groups $B R A F$-only and $B R A F+T E R T$, indicating that there was little difference in the transcriptional profile between the two groups (Fig. 4C). Only 12 genes showed a statistically significant difference but with modest changes (fold changes, 2.0-2.7): CILP, SFTPA1, SFTPA2, PODNL1, TSPAN11, CREB3L1, EGLN3, WISP2, GAS1, SLC38A3, MFAP5 and TFF3 (Supplementary Table 8). In the analysis of the SNU dataset, a similar pattern of transcriptional changes was observed, and there were no DEGs between groups BRAF-only and BRAF+TERT (Supplementary Fig. 4A, B and C). Notably, the sets of DEGs of groups $B R A F$-only and $B R A F+T E R T$ strongly overlapped (Fig. 4D). Nevertheless, when we compared the expression levels of common DEGs between groups no-mutation vs $B R A F$-only and no-mutation vs $B R A F+T E R T$, the expression of upregulated DEGs was increased further and that of downregulated DEGs was decreased further in the $B R A F+T E R T$ group in comparison with the $B R A F$-only group ( $P$ values according to the paired $t$ test: $3.88 \times 10^{-5}$ and $1.62 \times 10^{-11}$, respectively; Fig. $4 \mathrm{E}$ ), indicating that the degree of changes in the gene expression of $B R A F$-mutated PTCs was amplified by the addition of TERT promoter mutation. The results of functional enrichment analysis showed that the upregulated genes were related to the pathways including the extracellular matrix receptor interaction, focal adhesion, cytokine-cytokine receptor interaction and cell adhesion molecules, whereas downregulated genes were associated with metabolism-related pathways (Fig. 4F and Supplementary Table 9). Similar results were obtained in our database (Supplementary Fig. 4D, E and F). (c) 2019 Society for Endocrinology Published by Bioscientifica Ltd. Printed in Great Britain 

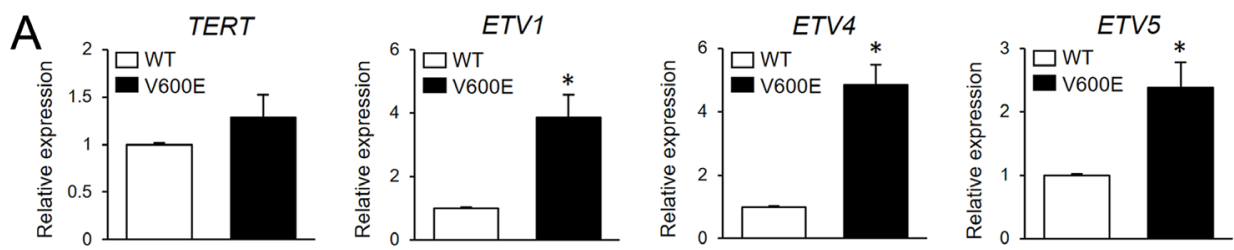

B
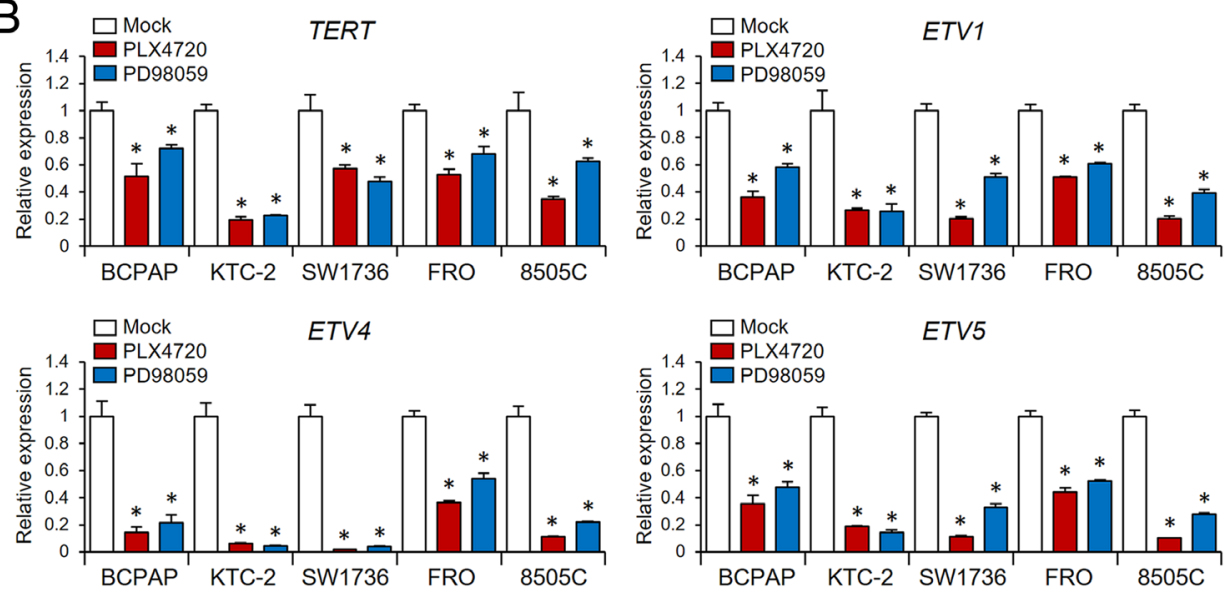

C
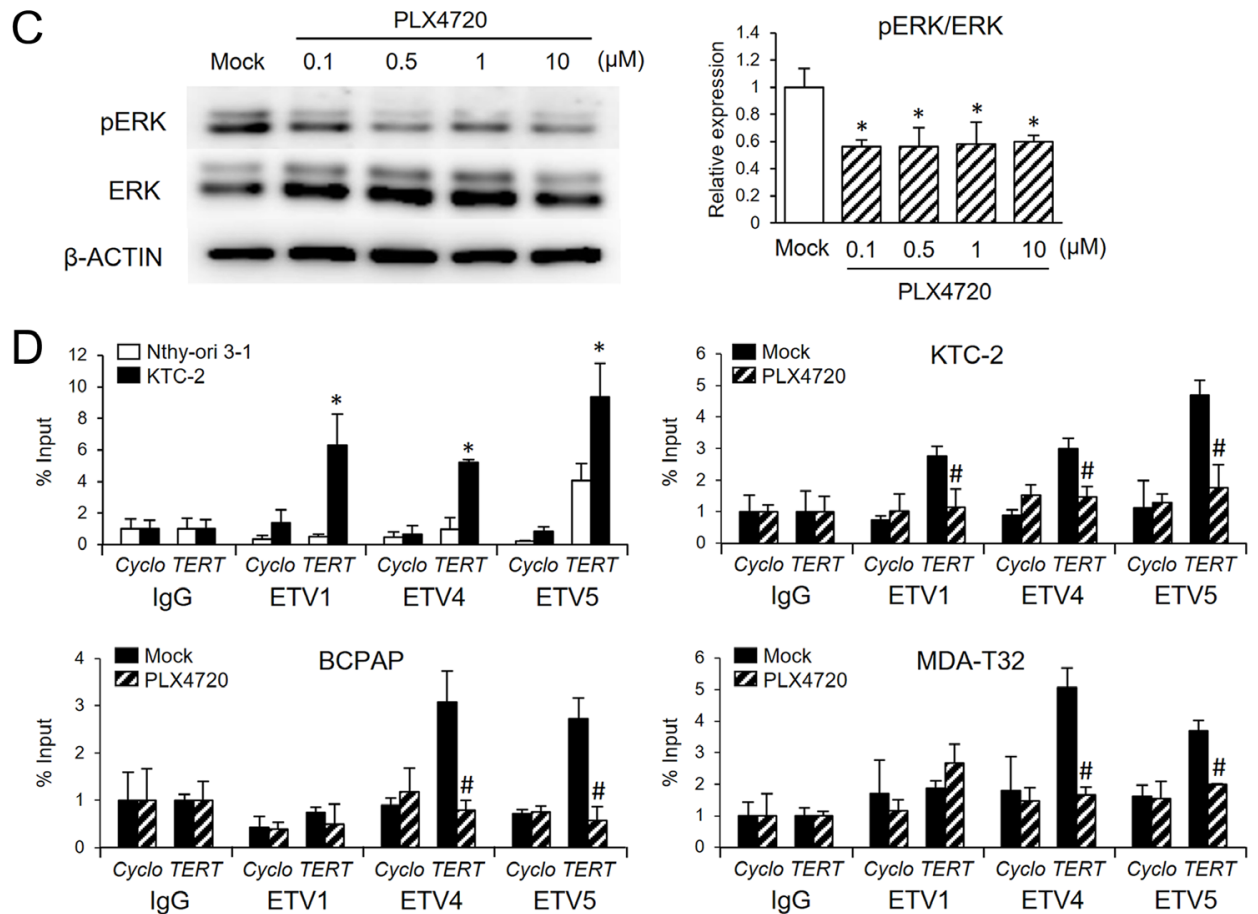

Figure 3

In vitro validation of changes in TERT and ETS expression by the BRAFV600E mutation and direct binding of ETS factors to the mutant TERT promoter. (A) In human thyroid cell lines expressing either wild-type BRAF (Nthy/WT) or mutant BRAF (Nthy/V600E), TERT, ETV1, ETV4 and ETV5 gene expression levels were quantified by RT-PCR. $* P<0.05$ vs. Nthy/WT. (B) BCPAP, KTC-2, SW1736, FRO, 8505C, the human thyroid cancer cell lines harboring both $B R A F v 600$ and TERT promoter mutations, were treated with PLX4720 (a BRAF inhibitor) or PD98059 (an ERK inhibitor), and TERT, ETV1, ETV4 and ETV5 gene expression levels were quantified by RT-PCR. ${ }^{*} P<0.05$ vs mock. (C) BCPAP cells were treated with PLX4720 $(0.1,0.5,1$ and $10 \mu \mathrm{M})$ and proteins were harvested from total cell lysates $24 \mathrm{~h}$ after treatment. Western blot analysis was performed with anti-pERK and anti-ERK. $* P<0.05$ vs mock. (D) Chromatin immunoprecipitation (ChIP) assay for ETV1, ETV4 and ETV5 occupancy at TERT promoter in Nthy-ori 3-1 cells without BRAFV600E and TERT promoter mutations and in KTC-2, BCPAP and MDA-T32 cells with both mutations. KTC-2, BCPAP and MDA-T32 cells were treated with PLX4720. The fold enrichment was the fold increase for the signal from each antibody-enriched chromatin relative to a control IgG. Cyclophilin (Cyclo) was used as negative control. All data are expressed as mean \pm S.D. $* P<0.05$ vs Nthy-ori $3-1, \# P<0.05$ vs mock. 


\section{Changes in the intracellular signaling pathways by TERT expression in PTCs harboring the BRAF ${ }^{600 E}$ mutation}

As shown in Fig. 1B and $\mathrm{D}$, in some tumors in the BRAFonly group, comparable expression of the TERT gene was observed relative to the $B R A F+T E R T$ group. This might be one of the reasons for the absence of DEGs between groups BRAF-only and BRAF+TERT (Fig. 4C). Then, we reclassified the samples according to the TERT expression status rather than TERT promoter mutation (Fig. 5A). The PTCs harboring the BRAFV600E mutation without or with TERT expression showed a significant change in transcriptional profiles compared to those without either BRAFVG00E mutation or TERT expression (Fig. 5B and C). The common pathways of DEGs between the BRAF mutation groups with and without TERT expression were almost identical to those between $B R A F$-only and $B R A F+T E R T$ mutation groups (Supplementary Fig. $5 \mathrm{~A}$ and B). Moreover, when we compared the expression levels of common DEGs between the two groups, as with the TERT promoter mutation, the degree of changes in the gene expression of BRAF-mutated PTCs was amplified by the addition of TERT expression (Supplementary Fig. 5C). On the other hand, in contrast to the TERT promoter mutation, a number of genes were activated by the presence of TERT expression in the BRAF-mutated tumors (Fig. 5D). Most of these upregulated genes belonged to pathways related to inflammatory cytokines or adhesion molecules (Fig. 5E and Supplementary Table 10). The expression of representative genes including $C D 28$, CTLA4 and VCAM1 was significantly increased by TERT expression (Fig. 5F). We validated these results on SNU data (Supplementary Fig. 6).
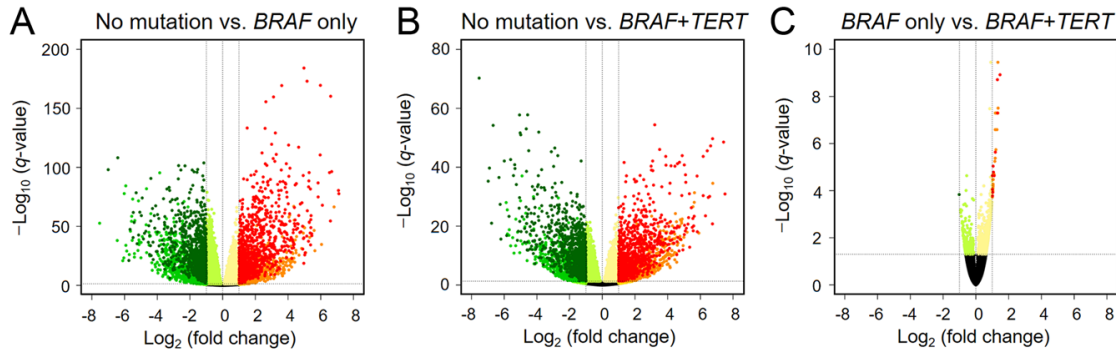

$\log _{2}$ (fold change) $\leq-1, q$-value $<0.05$, baseMean $\geq 100$ $\mathrm{Log}_{2}$ (fold change) $\leq-1, q$-value $<0.05$, baseMean $<100$ $-1<\log _{2}$ (fold change) $\leq-1, q$-value $<0.05$

- Log $_{2}$ (fold change) $\geq 1, q$-value $<0.05$, baseMean $\geq 100$ $\log _{2}$ (fold change) $\geq 1, q$-value $<0.05$, baseMean $<100$ $0<$ Log $_{2}$ (fold change) $\leq 1, q$-value $<0.05$

D

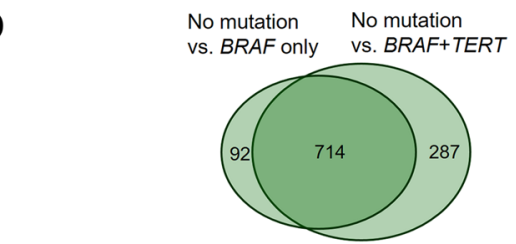

No mutation No mutation vs. BRAF only vs. BRAF+TERT

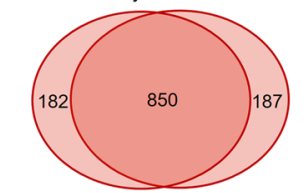

E

Down-regulated DEGs

Up-regulated DEGs

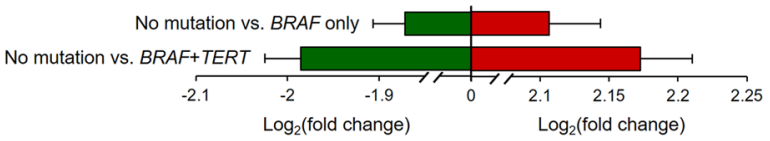

F

Down-regulated DEGs Up-regulated DEGs

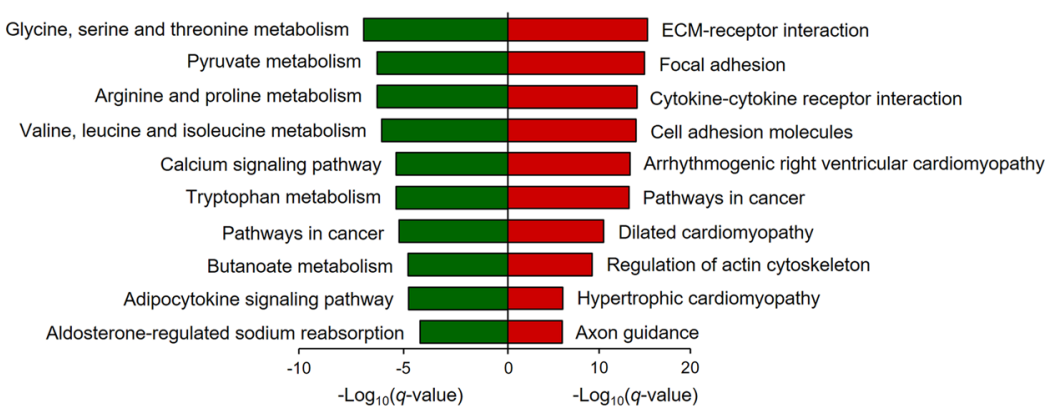

\section{Figure 4}

Transcriptional changes according to mutational status of the BRAFV600E and TERT promoter. (A, B and C) Volcano plots for DEGs according to mutational status of samples from TCGA database: no-mutation vs $B R A F$-only $(\mathrm{A})$, no-mutation vs $B R A F+T E R T(B)$, and $B R A F$-only vs $B R A F+T E R T$ (C). (D) Venn diagram for the overlapping downregulated (green) and upregulated (red) DEGs between groups no-mutation vs BRAF-only and no-mutation vs $B R A F+T E R T$. (E) The degree of changes in the gene expression of common DEGs, expressed as mean \pm standard error of the mean. $(F)$ The top 10 most significantly enriched molecular pathways of common DEGs. (c) 2019 Society for Endocrinology Published by Bioscientifica Ltd. Printed in Great Britain 


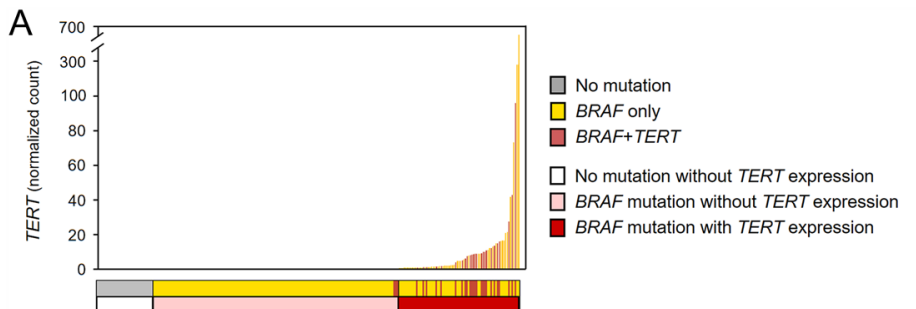

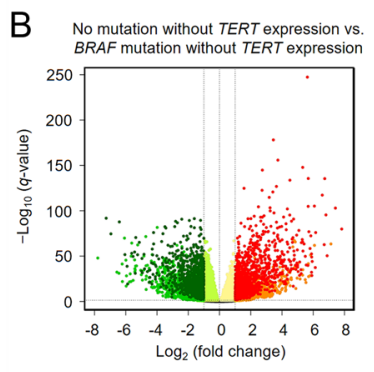
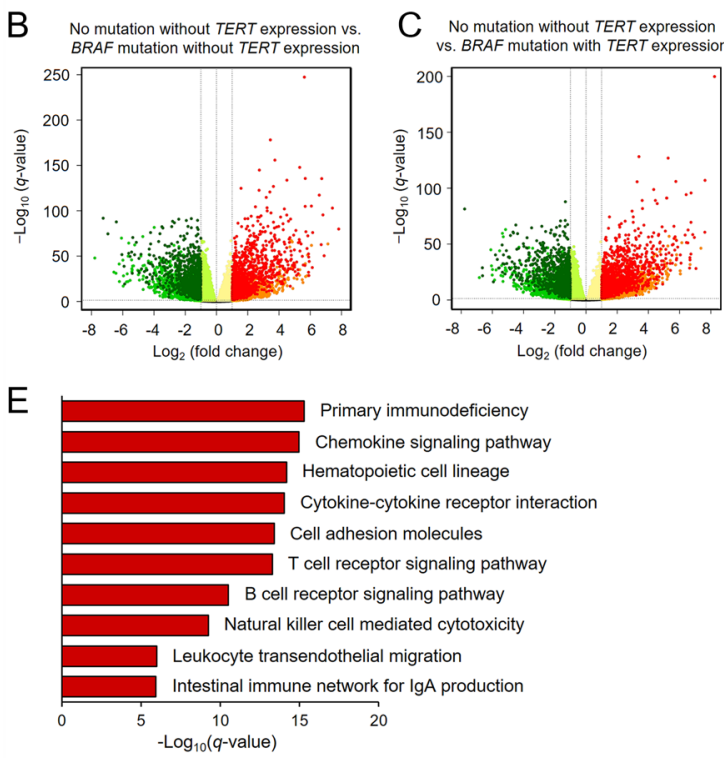

F
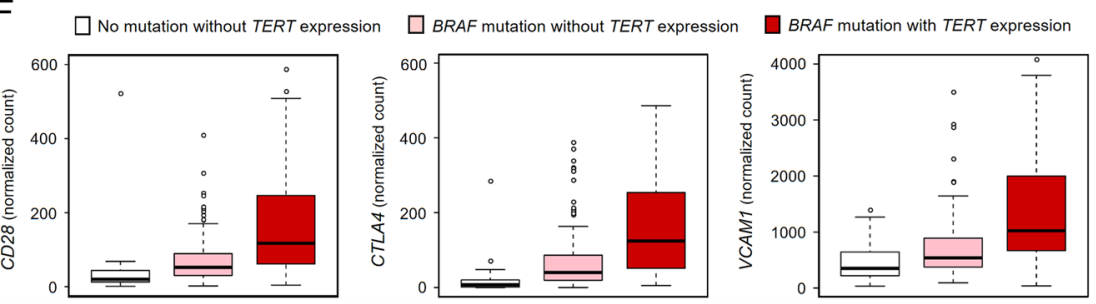

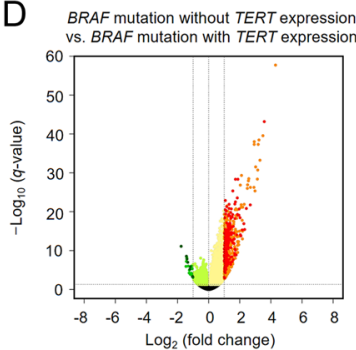

\section{Figure 5}

Transcriptional changes by TERT expression in addition to the BRAFV600E mutation. (A) Each column represents the TERT mRNA expression level of an individual sample of TCGA database. (B, C and D) Volcano plots for DEGs according to BRAFV600E mutation and TERT mRNA expression: no mutation without TERT expression vS BRAFV600E mutation without TERT expression (B), no mutation without TERT expression vs BRAFV600E mutation with TERT expression (C), and BRAFV600E mutation without TERT expression vS BRAFV600E mutation without TERT expression (D). (E) The top 10 most significantly enriched molecular pathways of upregulated DEGs in BRAF-mutated PTCs with TERT expression compared to those without TERT expression. (F) Median expression levels of genes CD28, CTLA4 and VCAM1, which were upregulated DEGs in BRAF-mutated PTCS with TERT expression compared to those without TERT expression.

\section{Discussion}

The clinicopathological outcomes of BRAF-mutated PTCs clearly became much more aggressive when they had a coexisting TERT promoter mutation. From the analyses of TCGA and SNU RNA sequencing data and in vitro experiments, we could confirm that TERT mRNA expression was increased by addition of the BRAFV600E mutation to the TERT promoter mutation. Furthermore, this increase was at least in part due to the upregulated expression of ETS, especially ETV1, ETV4 and ETV5 by the BRAFV600E mutation. On the other hand, coexisting mutations showed changes in almost the same intracellular signaling pathways as a BRAFV600E mutation alone but amplified the changes of the expression level of genes associated with altered pathways. Moreover, the inflammation and adhesion-related pathways were activated by addition of TERT expression in BRAF-mutated PTCs. From these results, we could obtain evidence for the possible molecular mechanism behind the synergistic effects of BRAFVG00E and TERT promoter mutation in thyroid cancer cells.

As for the mechanism, we confirmed that this genetic duet significantly increased TERT expression as compared with the expression in tumors harboring $B R A F^{V 600 E}$ or TERT promoter mutation alone. One previous study (Vinagre et al. 2013) also showed that the coexistence of BRAFV600E and TERT promoter mutations was associated with the highest levels of TERT mRNA expression although they included only three samples of thyroid cancer harboring both mutations. On the other hand, we confirmed that the expression of TERT was further upregulated by the
C 2019 Society for Endocrinology Published by Bioscientifica Ltd. Printed in Great Britain 
BRAFV600E mutation in PTC tissues with a mutant TERT promoter on a relatively large number of samples and validated this finding using the PTC cell lines harboring both mutations. Moreover, the similarity of pathways activated by BRAFV600E and both mutations may result in amplification of the pathways instead of activation of new pathways when they coexist. Commonly activated molecular pathways were found to be related to the signaling pathways or ontologies representing invasion, adhesion, carcinogenesis of thyroid nodules or cancer invasiveness (Nucera et al. 2011).

Moreover, among transcription factors which can control TERT promoter activity, the ETS expression showed the most significant difference, confirming the possibility that it plays an important role in the synergism between BRAF and TERT. Several research groups have reported that the major ETS transcription factors can actively bind to the mutated TERT promoter region because their binding motif was created by the mutation (Bell et al. 2015, Li et al. 2015). Although binding the core recognition sequence $5^{\prime}-\mathrm{GGA}(\mathrm{A} / \mathrm{T})-3^{\prime}$ is a common property of ETS transcription factors, a genome-wide analysis of all ETS family members has established some differences in DNA-binding preferences within this family. A previous study (Bell et al. 2015) revealed that the GABP transcription factor selectively binds to a mutant TERT promoter in glioblastoma samples and cell lines of neuroblastoma, melanoma and hepatocellular carcinoma. Moreover, Liu et al. (2018) recently reported the important findings that BRAFV600E/MAPK pathway increased GABPB expression and GABPA/B complex via FOS, and this complex selectively binds and upregulates mutant TERT promoter in human cancer, including thyroid cancer. Another recent study on melanoma (Vallarelli et al. 2016) showed that the ETS1 transcription factor, which was upregulated as a downstream target of the activated MAPK pathway, increases the transcriptional activity of TERT in melanoma cells harboring the TERT promoter mutation. However, in our study, GABP and ETS1 expression was not significantly affected by BRAFV600E, but we firstly showed ETV1, ETV4 and ETV5 were upregulated by the BRAFV600E/MAPK pathway activation in thyroid cancer. We demonstrated that these $B R A F^{V 600 E}$-induced ETS factors bound directly to the TERT promoter in a mutation-dependent manner, using ChIP assays. Furthermore, we confirmed the expression of ETV1, ETV4 and ETV5 was increased by
$B R A F$ overexpression and decreased by BRAF or ERK inhibition. The binding of these ETS factors to the mutant TERT promoter was also decreased by $B R A F$ inhibition, although there was no difference in ETV1 in several cell lines. However, one of the limitations of our study is that we did not show that each ETS factor directly induced TERT activation after binding to the mutant TERT promoter.

Some cases in the $B R A F$-only group exhibited TERT expression and this was associated with poorer clinicopathological outcomes. One of the potential mechanisms of TERT reactivation in this BRAF-only group is the TERT fusion gene which has been reported to markedly increase the expression of TERT in liposarcoma (Stransky et al. 2014) and kidney cancer (Karlsson et al. 2015). In addition, the methylation of TERT promoter also might be one of the mechanisms for regulating TERT expression. When we performed the TERT promoter methylation analysis using the DNA methylation array data from TCGA, there was a significant positive correlation between cg11625005, one of the CpG sites of the TERT promoter, and TERT mRNA expression levels in the $B R A F$-only group $(r=0.427, P<0.001)$. The presence of TERT expression in the BRAF-mutated tumors was associated with activation of inflammation and adhesion-related pathways, in agreement with one of the noncanonical roles of TERT such as activating the NF-кB signaling pathway (Low \& Tergaonkar 2013, Li \& Tergaonkar 2014). There was a report about the clinical importance of TERT expression in urothelial cancer, namely, that the TERT mRNA expression predicts clinical outcomes more accurately than does the TERT promoter mutation (Borah et al. 2015).

In conclusion, the mechanism of synergistic effects between BRAFV600E and TERT promoter mutations on aggressiveness of PTC may be explained by increased TERT expression, which may result from the $B R A F$-induced upregulation of several ETS transcription factors, especially ETV1, ETV4 and ETV5. Therefore, we can suggest that the $B R A F^{V 600 E}$ mutation upregulates the ETS transcription factors, and then, the TERT promoter mutation may turn on the switch of catastrophe by creating the ETS-binding sites and increasing TERT expression. Furthermore, the molecular pathways activated by the BRAFV600E mutation are further augmented by the TERT promoter mutation or TERT expression, which can synergistically enhance the effects on cancer invasiveness and progression. (c) 2019 Society for Endocrinology Published by Bioscientifica Ltd. Printed in Great Britain 


\section{Supplementary data}

This is linked to the online version of the paper at https://doi.org/10.1530/ ERC-17-0562.

\section{Declaration of interest}

The authors declare that there is no conflict of interest that could be perceived as prejudicing the impartiality of the research reported.

\section{Funding}

This research was supported by the Basic Science Research Program through the National Research Foundation of Korea, funded by the Ministry of Science, ICT \& Future Planning (NRF-2016R1A2B4012417).

\section{References}

Akincilar SC, Unal B \& Tergaonkar V 2016 Reactivation of telomerase in cancer. Cellular and Molecular Life Sciences 73 1659-1670. (https:// doi.org/10.1007/s00018-016-2146-9)

Bell RJ, Rube HT, Kreig A, Mancini A, Fouse SD, Nagarajan RP, Choi S, Hong C, He D, Pekmezci M, et al. 2015 Cancer. The transcription factor GABP selectively binds and activates the mutant tert promoter in cancer. Science 348 1036-1039. (https://doi.org/10.1126/science. aab0015)

Borah S, Xi L, Zaug AJ, Powell NM, Dancik GM, Cohen SB, Costello JC, Theodorescu D \& Cech TR 2015 Cancer. Tert promoter mutations and telomerase reactivation in urothelial cancer. Science $\mathbf{3 4 7}$ 1006-1010. (https://doi.org/10.1126/science.1260200)

Cancer Genome Atlas Research Network 2014 Integrated genomic characterization of papillary thyroid carcinoma. Cell 159 676-690. (https://doi.org/10.1016/j.cell.2014.09.050)

de Hoon MJ, Imoto S, Nolan J \& Miyano S 2004 Open source clustering software. Bioinformatics 20 1453-1454. (https://doi.org/10.1093/ bioinformatics/bth078)

Horn S, Figl A, Rachakonda PS, Fischer C, Sucker A, Gast A, Kadel S, Moll I, Nagore E, Hemminki K, et al. 2013 Tert promoter mutations in familial and sporadic melanoma. Science 339 959-961. (https:// doi.org/10.1126/science.1230062)

Huang FW, Hodis E, Xu MJ, Kryukov GV, Chin L \& Garraway LA 2013 Highly recurrent tert promoter mutations in human melanoma. Science 339 957-959. (https://doi.org/10.1126/ science.1229259)

Jeong YS, Kim D, Lee YS, Kim HJ, Han JY, Im SS, Chong HK, Kwon JK, Cho YH, Kim WK, et al. 2011 Integrated expression profiling and genome-wide analysis of ChREBP targets reveals the dual role for ChREBP in glucose-regulated gene expression. PLoS ONE 6 e22544. (https://doi.org/10.1371/journal.pone.0022544)

Karlsson J, Lilljebjorn H, Holmquist Mengelbier L, Valind A, Rissler M, Ora I, Fioretos T \& Gisselsson D 2015 Activation of human telomerase reverse transcriptase through gene fusion in clear cell sarcoma of the kidney. Cancer Letters 357 498-501. (https://doi. org/10.1016/j.canlet.2014.11.057)

Kim BA, Jee HG, Yi JW, Kim SJ, Chai YJ, Choi JY \& Lee KE 2017 Expression profiling of a human thyroid cell line stably expressing the BRAFV600E mutation. Cancer Genomics and Proteomics 14 53-67. (https://doi.org/10.21873/cgp.20018)
Landa I, Ibrahimpasic T, Boucai L, Sinha R, Knauf JA, Shah RH, Dogan S, Ricarte-Filho JC, Krishnamoorthy GP, Xu B, et al. 2016 Genomic and transcriptomic hallmarks of poorly differentiated and anaplastic thyroid cancers. Journal of Clinical Investigation 126 1052-1066. (https://doi.org/10.1172/JCI85271)

Li Y \& Tergaonkar V 2014 Noncanonical functions of telomerase: implications in telomerase-targeted cancer therapies. Cancer Research 74 1639-1644. (https://doi.org/10.1158/0008-5472. CAN-13-3568)

Li Y, Zhou QL, Sun W, Chandrasekharan P, Cheng HS, Ying Z, Lakshmanan M, Raju A, Tenen DG, Cheng SY, et al. 2015 Noncanonical NF-kappaB signalling and ETS1/2 cooperatively drive C250T mutant tert promoter activation. Nature Cell Biology 17 1327-1338. (https://doi.org/10.1038/ncb3240)

Liu X, Bishop J, Shan Y, Pai S, Liu D, Murugan AK, Sun H, El-Naggar AK \& Xing M 2013 Highly prevalent tert promoter mutations in aggressive thyroid cancers. Endocrine-Related Cancer 20 603-610. (https://doi.org/10.1530/ERC-13-0210)

Liu C, Liu Z, Chen T, Zeng W, Guo Y \& Huang T 2016 Tert promoter mutation and its association with clinicopathological features and prognosis of papillary thyroid cancer: a meta-analysis. Scientific Reports 6 36990. (https://doi.org/10.1038/srep36990)

Liu RY, Bishop J, Zhu GW, Zhang T, Ladenson PW \& Xing MZ 2017 Mortality risk stratification by combining BRAF V600E and tert promoter mutations in papillary thyroid cancer genetic duet of BRAF and tert promoter mutations in thyroid cancer mortality. JAMA Oncology 3 202-208. (https://doi.org/10.1001/ jamaoncol.2016.3288)

Liu R, Zhang T, Zhu G \& Xing M 2018 Regulation of mutant tert by BRAF V600E/MAP kinase pathway through FOS/GABP in human cancer. Nature Communications 9 579. (https://doi.org/10.1038/ s41467-018-03033-1)

Love MI, Huber W \& Anders S 2014 Moderated estimation of fold change and dispersion for RNA-seq data with DESeq2. Genome Biology 15 550. (https://doi.org/10.1186/s13059-014-0550-8)

Low KC \& Tergaonkar V 2013 Telomerase: central regulator of all of the hallmarks of cancer. Trends in Biochemical Sciences 38 426-434. (https://doi.org/10.1016/j.tibs.2013.07.001)

Moon S, Song YS, Kim YA, Lim JA, Cho SW, Moon JH, Hahn S, Park DJ \& Park YJ 2017 Effects of coexistent BRAFV600E and tert promoter mutations on poor clinical outcomes in papillary thyroid cancer: a meta-analysis. Thyroid 27 651-660. (https://doi.org/10.1089/ thy.2016.0350)

Nucera C, Lawler J \& Parangi S 2011 BRAF(V600E) and microenvironment in thyroid cancer: a functional link to drive cancer progression. Cancer Research 71 2417-2422. (https://doi. org/10.1158/0008-5472.CAN-10-3844)

Ogata H, Goto S, Sato K, Fujibuchi W, Bono H \& Kanehisa M 1999 KEGG: Kyoto encyclopedia of genes and genomes. Nucleic Acids Research 27 29-34. (https://doi.org/10.1093/nar/27.1.29)

Ramlee MK, Wang J, Toh WX \& Li S 2016 Transcription regulation of the human telomerase reverse transcriptase (hTERT) gene. Genes 7 E50. (https://doi.org/10.3390/genes7080050)

Song YS, Lim JA \& Park YJ 2015 Mutation profile of well-differentiated thyroid cancer in Asians. Endocrinology and Metabolism 30 252-262. (https://doi.org/10.3803/EnM.2015.30.3.252)

Song YS, Lim JA, Choi H, Won JK, Moon JH, Cho SW, Lee KE, Park YJ, Yi KH, Park DJ, et al. 2016 Prognostic effects of tert promoter mutations are enhanced by coexistence with BRAF or RAS mutations and strengthen the risk prediction by the ATA or TNM staging system in differentiated thyroid cancer patients. Cancer $\mathbf{1 2 2}$ 1370-1379. (https://doi.org/10.1002/cncr.29934) https://erc.bioscientifica.com https://doi.org/10.1530/ERC-17-0562 (c) 2019 Society for Endocrinology Published by Bioscientifica Ltd. Printed in Great Britain 
Stransky N, Cerami E, Schalm S, Kim JL \& Lengauer C 2014 The landscape of kinase fusions in cancer. Nature Communications 54846. (https://doi.org/10.1038/ncomms5846)

Vallarelli AF, Rachakonda PS, Andre J, Heidenreich B, Riffaud L, Bensussan A, Kumar R \& Dumaz N 2016 Tert promoter mutations in melanoma render tert expression dependent on MAPK pathway activation. Oncotarget 7 53127-53136. (https://doi.org/10.18632/ oncotarget.10634)

Vinagre J, Almeida A, Populo H, Batista R, Lyra J, Pinto V, Coelho R, Celestino R, Prazeres H, Lima L, et al. 2013 Frequency of tert promoter mutations in human cancers. Nature Communications 4 2185. (https://doi.org/10.1038/ncomms3185)
Xing M 2010 Prognostic utility of BRAF mutation in papillary thyroid cancer. Molecular and Cellular Endocrinology 321 86-93. (https://doi. org/10.1016/j.mce.2009.10.012)

Xing M, Liu R, Liu X, Murugan AK, Zhu G, Zeiger MA, Pai S \& Bishop J 2014 BRAF V600E and tert promoter mutations cooperatively identify the most aggressive papillary thyroid cancer with highest recurrence. Journal of Clinical Oncology 32 2718-2726. (https://doi. org/10.1200/JCO.2014.55.5094)

Yoo SK, Lee S, Kim SJ, Jee HG, Kim BA, Cho H, Song YS, Cho SW, Won JK, Shin JY, et al. 2016 Comprehensive analysis of the transcriptional and mutational landscape of follicular and papillary thyroid cancers. PLOS Genetics 12 e1006239. (https://doi.org/10.1371/journal.pgen.1006239)

Received in final form 26 March 2019

Accepted 17 April 2019

Accepted Preprint published online 17 April 2019 (c) 2019 Society for Endocrinology Published by Bioscientifica Ltd. Printed in Great Britain 